\title{
IncRNA XIST knockdown suppresses cell proliferation and promotes apoptosis in diabetic cataracts through the miR-34a/SMAD2 axis
}

\author{
CHAO WANG ${ }^{1}$, RUILING ZHAO ${ }^{2}$ and SUHONG ZHANG ${ }^{1}$ \\ ${ }^{1}$ Department of Ophthalmology, Shandong Zaozhuang Municipal Hospital, Zaozhuang, Shandong 277100; \\ ${ }^{2}$ Department of Ophthalmology, Shandong Tengzhou Central People's Hospital, Zaozhuang, Shandong, 277599, P.R. China
}

Received February 1, 2021; Accepted July 22, 2021

DOI: $10.3892 / \mathrm{mmr} .2021 .12523$

\begin{abstract}
According to emerging evidence, long non-coding RNAs (lncRNAs) play critical roles in diabetes. The aim of the present study was to investigate the role and mechanism of X-inactive specific transcript (XIST) in cell proliferation, migration and apoptosis in diabetic cataracts (DC). SRA01/04 lens epithelial cells were treated with high glucose (HG). The levels of XIST, microRNA (miR)-34a and SMAD family member 2 (SMAD2) were examined via reverse transcription-quantitative PCR. MTT, Transwell, wound healing and TUNEL assays were performed to examine cell proliferation, invasion, migration and apoptosis, respectively. The interaction between miR-34a and XIST or SMAD2 was verified by luciferase reporter assay. It was found that the expression of XIST was increased and that of miR-34a was decreased in DC tissues and HG-treated SRA01/04 cells. XIST knockdown or miR-34a overexpression attenuated cell proliferation and migration, and induced apoptosis in HG-treated SRA01/04 cells. XIST targeted miR-34a and regulated DC progression through miR-34a. SMAD2 was identified as a target gene of miR-34a and was positively modulated by XIST. XIST knockdown inhibited cell proliferation and migration, and accelerated apoptosis in HG-stimulated SRA01/04 cells, and these effects were abrogated by SMAD2 overexpression. In conclusion, XIST promoted cell proliferation, migration and invasion, and inhibited apoptosis, through the miR-34a/SMAD2 axis in DC.
\end{abstract}

\section{Introduction}

Diabetes mellitus causes several complications, including the formation and occurrence of cataracts (1). Despite the

Correspondence to: Professor Suhong Zhang, Department of Ophthalmology, Shandong Zaozhuang Municipal Hospital, 41 Longtou Road, Zaozhuang, Shandong 277100, P.R. China E-mail: suhong_zhang1@163.com

Key words: X-inactive specific transcript, microRNA-34a, SMAD family member 2 , lens epithelial cells, diabetic cataracts successful surgical replacement of cataracts with intraocular lenses, cataracts remain one of the leading causes of visual impairment and blindness worldwide (2). Diabetic cataracts (DC) characterized by high blood glucose levels usually occur earlier and progress faster than cataract $(3,4)$. Human lens epithelial cells (HLECs) have been reported to play essential roles in ocular health, as well as several diseases, including DC (5). The pathogenesis of DC is a multifactorial process, and gene alterations that are associated with the proliferation, differentiation and epithelial-to-mesenchymal transition (EMT) of LECs may lead to the occurrence of cataract (6). It is therefore crucial to explore the mechanism underlying LEC proliferation in DC.

Long non-coding RNAs (lncRNAs) are a group of non-coding RNAs of $>200 \mathrm{nts}$ in length that lack proteinencoding potential (7). In addition, IncRNAs have been reported to participate in DC development. For example, lncRNA metastasis-associated lung adenocarcinoma transcript 1 has been shown to facilitate the apoptosis and oxidative stress of HLECs through the p38 MAPK pathway in DC (8). Sp1 transcription factor-mediated lncRNA Pvtl oncogene was shown to regulate LEC viability and apoptosis in DC through the microRNA (miRNA/miR)-214-3p/MMP2 axis (9). Furthermore, lncRNA X-inactive specific transcript (XIST) has been found to be associated with diabetic complications, such as diabetic nephropathy and retinopathy $(10,11)$. However, the biological role of XIST during DC remains unclear.

miRNAs are small RNAs that can target the 3'-untranslated region (UTR) of mRNAs to modulate gene transcription (12). miRNA dysregulation has been observed in multiple diseases, including DC. For example, miR-30a was shown to suppress autophagy by targeting Beclin 1 in human DC (13). miR-211 was found to promote apoptosis and repress proliferation of LECs in DC mice by targeting SIRT1 (14). In addition, miR-34a inhibited LEC viability and induced apoptosis by targeting E2F3 (15). Furthermore, SMAD family member 2 (SMAD2) has been reported to play a crucial role in posterior capsular opacification (16). Nevertheless, the regulatory mechanisms of miR-34a and SMAD2 during DC remain largely unknown. In the present study, SRA01/04 cells were stimulated by high glucose (HG) to establish a DC model, and the biological effects of XIST on DC were then investigated. 


\section{Materials and methods}

Samples. A total of 32 posterior capsular tissue samples from patients with DC (18 men, 14 women; age range, 52-60 years) and paired normal posterior capsular tissue samples without DC (17 men, 15 women; age range, 48-59 years) were obtained from the Shandong Zaozhuang Municipal Hospital (Zaozhuang, China). The tissues were rapidly frozen in liquid nitrogen at $-80^{\circ} \mathrm{C}$ prior to use. This study was approved by the Ethics Committee of Shandong Zaozhuang Municipal Hospital and all participants signed informed consent forms prior to surgery. Patients diagnosed with DC and provided informed consent were included in this study. Patients with complex cataracts with high myopia, ocular trauma and ocular inflammation were excluded.

Cell culture and HG treatment. SRA01/04 HLECs were purchased from BeNa Culture Collection (Beijing Beina Chuanglian Institute of Biotechnology). The cells were incubated at $37^{\circ} \mathrm{C}$ with $5 \% \mathrm{CO}_{2}$ in DMEM (Thermo Fisher Scientific, Inc.) containing 10\% FBS (Thermo Fisher Scientific, Inc.) and $1 \%$ penicillin/streptomycin. To establish the DC cell model, SRA01/04 cells were maintained in medium containing $\mathrm{HG}(25 \mathrm{mM})$ for $24 \mathrm{~h}$ at $37^{\circ} \mathrm{C}$, and cells in normal glucose (NG; $5.5 \mathrm{mM}$ ) were used as the control.

Cell transfection. Small hairpin (sh)RNA against XIST (shXIST, 5'-GUGCGUACAGUGCUGUACAGCAU-3') and its negative control (NC; shNC, 5'-UACGCUCAGCAUGUGUCA CUC-3'), miR-34a mimics (5'-UCGUUCGUGAGCACUUGC GACG-3'), NC mimics (5'-UCGUCGGAUCGACUGAGA UCU-3'), miR-34a inhibitors (5'-AGCCUUGCUGCAGGU GCGCAU-3') and NC inhibitors (5'-UGCCUUACUGACGGU CGGAGA-3') were obtained from Shanghai GenePharma Co., Ltd. pcDNA3.1 vector (Thermo Fisher Scientific, Inc.) was used to construct a XIST and SMAD2 overexpression vector. SRA01/04 cells $\left(1 \times 10^{5}\right)$ were transfected with $50 \mathrm{nM}$ shXIST, $50 \mathrm{nM}$ shNC, $50 \mathrm{nM}$ miR-34a mimics, $50 \mathrm{nM}$ miR-34a inhibitors, $50 \mathrm{nM}$ NC mimics or $50 \mathrm{nM}$ NC inhibitors using Lipofectamine ${ }^{\circledR} 2000$ (Thermo Fisher Scientific, Inc.) at room temperature for $\sim 30 \mathrm{~min}$, according to the manufacturer's protocol. After $48 \mathrm{~h}$ of transfection, the transfected cells were used for subsequent experiments.

Reverse transcription-quantitative $(R T-q) P C R$. TRIzol ${ }^{\circledR}$ reagent (Thermo Fisher Scientific, Inc.) was used to isolate total RNA from the cultured cells and tissue samples. PrimeScript ${ }^{\mathrm{TM}}$ RT reagent kit (Takara Bio, Inc.) was used for cDNA generation of XIST and SMAD2, and TaqMan ${ }^{\mathrm{TM}}$ MicroRNA Reverse Transcription kit (Thermo Fisher Scientific, Inc.) was used for cDNA generation of miR-34a, according to the manufacturer's protocol. qPCR was performed on the ABI 7900 Detection System (Thermo Fisher Scientific, Inc.) using the SYBR-Green PCR Master Mix kit (Thermo Fisher Scientific, Inc.). The $2^{-\triangle \Delta C q}$ method (17) was used to calculate the relative expression. The relative miR-34a expression was normalized to U6. GAPDH served as the control for XIST and SMAD2 expression. The thermocycling conditions were as follows: Pre-denaturation at $95^{\circ} \mathrm{C}$ for $1 \mathrm{~min}$, followed by 40 cycles of $95^{\circ} \mathrm{C}$ for $15 \mathrm{sec}, 60^{\circ} \mathrm{C}$ for $30 \mathrm{sec}$ and $72^{\circ} \mathrm{C}$ for $30 \mathrm{sec}$. The following primers were used: XIST, forward 5'-TCAGCCCAT CAGTCCAAGATC-3' and reverse 5'-CCTAGTTCAGGC CTGCTTTTCAT-3'; miR-34a, forward 5'-ACCCAGTGCGAT TTGTCA-3' and reverse 5'-ACTGTACTGGAAGATGGA CC-3'; SMAD2, forward 5'-TCCTACTACCGCCTCACA-3' and reverse 5'-ACСТCСТCСТCСТССТCT-3'; GAPDH, forward 5'-TCGACAGTCAGCCGCATCTTCTTT-3' and reverse 5'-ACCAAATCCGTTGACTCCGACCTT-3'; and U6, forward 5'-GCTTCGGCAGCACATATACTAAAAT-3' and reverse 5'-CGCTTCACGAATTTGCGTGTCA-3'.

Western blot analysis. Total protein was extracted from cells using RIPA lysis buffer (Sangon Biotech Co., Ltd.). Protein concentration was detected using a BCA assay kit (Sangon Biotech Co., Ltd.). Next, the proteins $(20 \mu \mathrm{g})$ were separated via $10 \%$ SDS-PAGE and transferred to PVDF membranes (MilliporeSigma). After blocking with 5\% non-fat milk for $2 \mathrm{~h}$, the membrane was incubated with primary antibodies against SMAD2 (1:1,000; cat. no. ab40855; Abcam) and GADPH (1:1,000; cat. no. ab9485; Abcam) at $4^{\circ} \mathrm{C}$. Subsequently, the membranes were washed with $0.1 \%$ Tween-20 and incubated with a HRP-conjugated secondary antibody $(1: 1,000$; cat. no. ab205718; Abcam) for $2 \mathrm{~h}$ at room temperature. Finally, protein bands were visualized using an ECL reagent (Beyotime Institute of Biotechnology).

MTT assay. Cell proliferation was assessed using MTT assay. Transfected SRA01/04 cells ( $1 \times 10^{5}$ cells/well) were cultured in 96-well plates and incubated for $0,24,48$ or $72 \mathrm{~h}$. MTT ( $5 \mathrm{mg} / \mathrm{ml}$ ) was then added for $4 \mathrm{~h}$ at $37^{\circ} \mathrm{C}$. Next, $150 \mu 1 \mathrm{DMSO}$ (Thermo Fisher Scientific, Inc.) was added to each well, and the optical density $490 \mathrm{~nm}$ value was measured using a microplate reader (BioTek Instruments, Inc.).

Transwell assay. SRA01/04 cell invasion was assessed by Transwell assay $(8.0-\mu \mathrm{m}$ pore size; MilliporeSigma) with Matrigel (Corning Inc.) precoating at room temperature for $1 \mathrm{~h}$. SRA01/04 cells $\left(2 \times 10^{5}\right.$ per well) were plated in the upper Transwell chamber in serum-free DMEM. Next, $500 \mu \mathrm{l}$ DMEM (10\% FBS) was plated in the lower chambers. Following incubation for $48 \mathrm{~h}$ at $37^{\circ} \mathrm{C}$, invading cells were stained with $0.1 \%$ crystal violet for $20 \mathrm{~min}$ at room temperature and counted under a light microscope (magnification, x100; Olympus Corporation).

Wound healing assay. A total of 5x105 SRA01/04 cells were cultured in DMEM supplemented without serum in 6-well plates and allowed to reach $70 \%$ confluence for the wound healing assay. A $200-\mu 1$ pipette tip was used to generate artificial scratches. Images of migrated cells were captured at 0 and $24 \mathrm{~h}$ using a microscope (magnification, x100; Leica DMI4000B; Leica Microsystems, Ltd.).

TUNEL assay. TUNEL Apoptosis Assay kit (Roche Diagnostics $\mathrm{GmbH}$ ) was used to assess cell apoptosis. Following fixation in $4 \%$ paraformaldehyde for $1 \mathrm{~h}$ at $4^{\circ} \mathrm{C}$, $1 \times 10^{5}$ SRA01/04 cells were cultured with TUNEL reaction mixture (Roche Diagnostics $\mathrm{GmbH}$ ) for $1 \mathrm{~h}$ at room temperature. Nuclear staining with DAPI was then performed for $15 \mathrm{~min}$ at room temperature. The TUNEL-positive cells were 


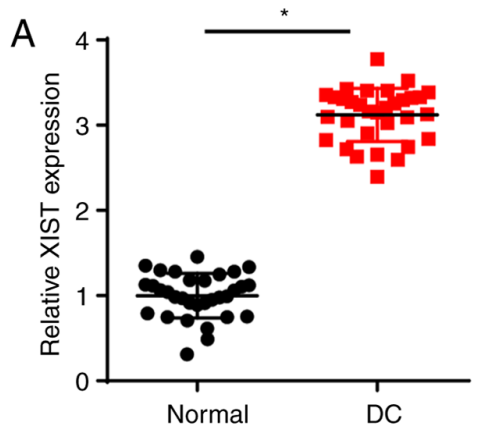

D

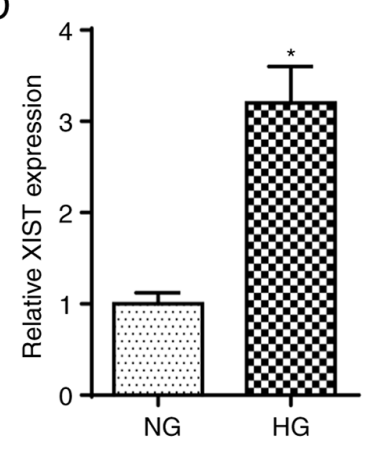

$\mathrm{B}$

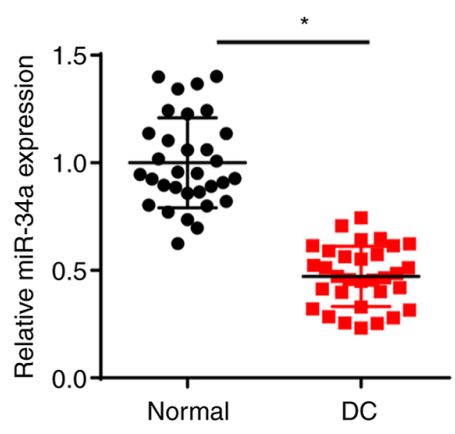

C

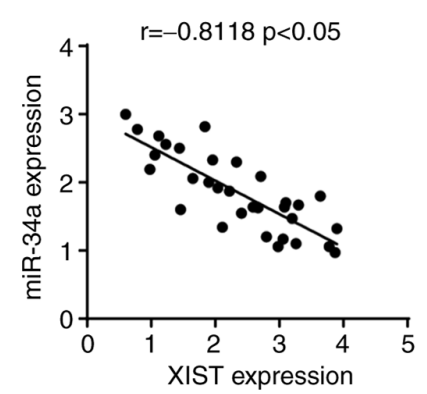

$\mathrm{E}$

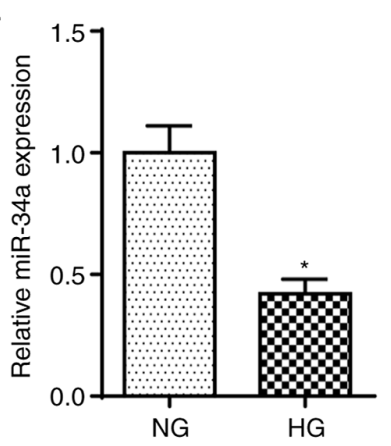

Figure 1. XIST is upregulated and miR-34a is downregulated in DC tissues and cells. (A and B) RT-qPCR showed the relative XIST and miR-34a expression in DC tissues and normal samples. "P $<0.05$. (C) Pearson's correlation analysis was used to determine the correlation between the miR-34a expression and XIST in DC tissues. (D and E) RT-qPCR showed the relative XIST and miR-34a expression in SRA01/04 cells treated with HG. $\mathrm{n}=3$. ${ }^{*} \mathrm{P}<0.05$ vs. NG. XIST, X-inactive specific transcript; miR, microRNA; DC, diabetic cataracts; RT-qPCR, reverse transcription-quantitative PCR; NG, normal glucose; HG, high glucose.

counted in five randomly selected fields under a fluorescence microscope (magnification, x200; Nikon Corporation).

Dual-luciferase reporter assay. The potential binding sites of miR-34a and XIST or SMAD2 were predicted using starBase 2.0 (http://starbase.sysu.edu.cn), as previously described $(18,19)$. The wild-type (wt) or mutant (mut) 3'-UTR sequences of XIST or SMAD2 were cloned into the pmirGLO vector (Promega Corporation). Site-directed mutagenesis was used to create the mut 3'-UTR sequence. The pmirGLO-XIST-wt or XIST-mut and pmirGLO-SMAD2-wt or SMAD2-mut reporter vector were co-transfected with miR-34a mimics and NC mimics into SRA01/04 cells using Lipofectamine ${ }^{\circledR} 2000$ (Thermo Fisher Scientific, Inc.). Following a 48-h incubation, luciferase activity was measured by Dual-Luciferase Reporter Assay System (Promega Corporation). Firefly luciferase activity was normalized to Renilla (Promega Corporation) luciferase gene activity.

RNA immunoprecipitation (RIP) assay. RIP assay was performed using the Magna RIP RNA-Binding Protein Immunoprecipitation kit (MilliporeSigma). SRA01/04 cells were lysed in RIP lysis buffer (MilliporeSigma). The obtained cell lysate $(100 \mu \mathrm{l})$ was centrifuged at $40,000 \mathrm{x} \mathrm{g}$ at $4^{\circ} \mathrm{C}$ for 10 min and incubated with $50 \mu \mathrm{l} / \mathrm{G}$ magnetic beads conjugated with $5 \mu \mathrm{g}$ anti- $\mathrm{AGO}_{2}(5 \mu \mathrm{g}$; cat. no. ab32381; Abcam) or $5 \mu \mathrm{g}$ anti-IgG antibodies (cat. no. ab172730; Abcam) for $1 \mathrm{~h}$ at $4^{\circ} \mathrm{C}$. Subsequently, the beads were washed three times using RIP Wash Buffer. The beads were then incubated with proteinase $\mathrm{K}$ buffer at $55^{\circ} \mathrm{C}$ for $30 \mathrm{~min}$ to digest the protein. Finally, XIST and miR-34a enrichment was measured via RT-qPCR.
Statistical analysis. Data are expressed as the mean \pm SD and analysis was performed using SPSS 17.0 (SPSS, Inc.). Each experiment was performed in triplicate. The correlation between miR-34a and XIST or SMAD2 was assessed by Pearson's correlation analysis. Comparisons between two groups or among multiple groups were assessed using an unpaired Student's t-test or one-way ANOVA followed by Tukey's post hoc test. $\mathrm{P}<0.05$ was considered to indicate a statistically significant difference.

\section{Results}

XIST is upregulated and miR-34a is downregulated in DC tissues and cells. First, the levels of XIST and miR-34a were detected in DC tissues by RT-qPCR. As shown in Fig. 1A and B, the expression of XIST was increased and that of miR-34a was reduced in DC samples. In addition, an inverse correlation between the miR-34a and XIST levels was observed in DC tissues (Fig. 1C). In addition, RT-qPCR analysis determined that the XIST expression was significantly elevated and miR-34a abundance was reduced in SRA01/04 cells treated with HG compared with the NG group (Fig. 1D and E). The data suggested that XIST was significantly upregulated and miR-34a was significantly downregulated in DC.

XIST depletion inhibits DC development in HG-treated LECs. To explore the role of XIST in DC, SRA01/04 cells were transfected with shXIST and shNC prior to HG treatment. RT-qPCR analysis revealed that XIST knockdown decreased the expression of XIST in SRA01/04 cells (Fig. 2A). Next, MTT, wound healing and Transwell assays revealed that XIST knockdown suppressed the proliferation, migration and 

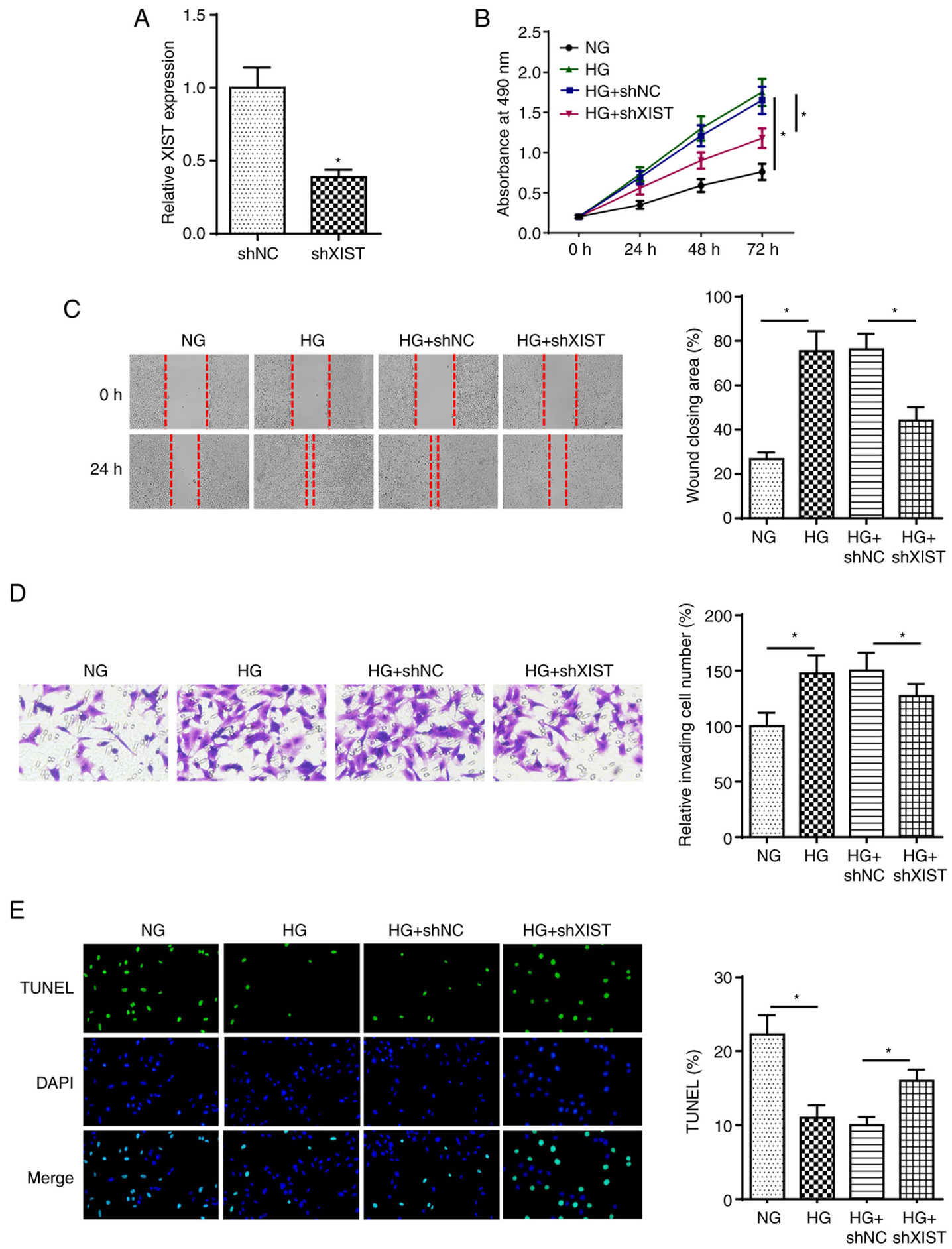

Figure 2. XIST depletion inhibits diabetic cataracts development in HG-induced lens epithelial cells. (A) Reverse transcription-quantitative PCR was used to determine the relative XIST expression in SRA01/04 transfected with shXIST. "P<0.05 vs. shNC. (B) MTT, (C) wound healing and (D) Transwell assays were used to analyze the proliferation, migration and invasion of SRA01/04 cells transfected with shXIST or shNC after treatment of HG. (E) TUNEL assay was used to determine the apoptosis in SRA01/04 cells transfected with shXIST or shNC after treatment of HG. $\mathrm{n}=3$. " $\mathrm{P}<0.05$. XIST, X-inactive specific transcript; sh, short hairpin RNA; NG, normal glucose; HG, high glucose; NC, negative control.

invasion of SRA01/04 cells treated by HG (Fig. 2B-D). XIST depletion induced the apoptosis of HG-stimulated SRA01/04 cells (Fig. 2E). These results indicated that XIST regulated HG-treated LEC proliferation, metastasis and apoptosis in DC.

miR-34a overexpression reduces $H G$-induced decrease in proliferation, migration and invasion in LECs. To investigate the effect of miR-34a on DC, SRA01/04 cells were transfected with miR-34a mimics. As shown in Fig. 3A, miR-34a was highly expressed following miR-34a overexpression. Furthermore, HG stimulation increased SRA01/04 cell proliferation, migration and invasion, while miR-34a overexpression reversed these effects (Fig. 3B-D). In addition, the HG treatment-induced decrease in cell apoptosis was rescued by miR-34a overexpression (Fig. 3E). These findings indicated that miR-34a played an essential role in HG-treated LECs. 
A

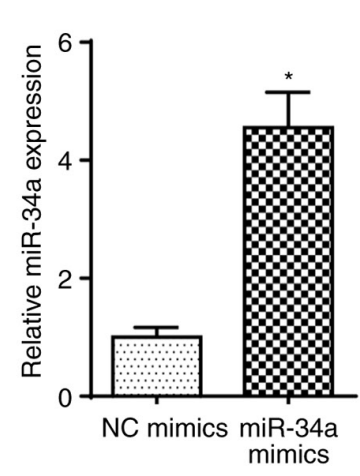

$B \quad \rightarrow N G$

$\rightarrow \mathrm{HG}+\mathrm{NC}$ mimic

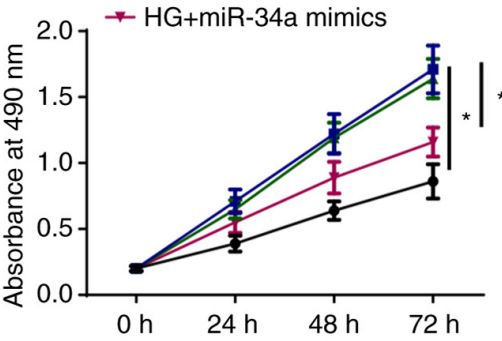

C
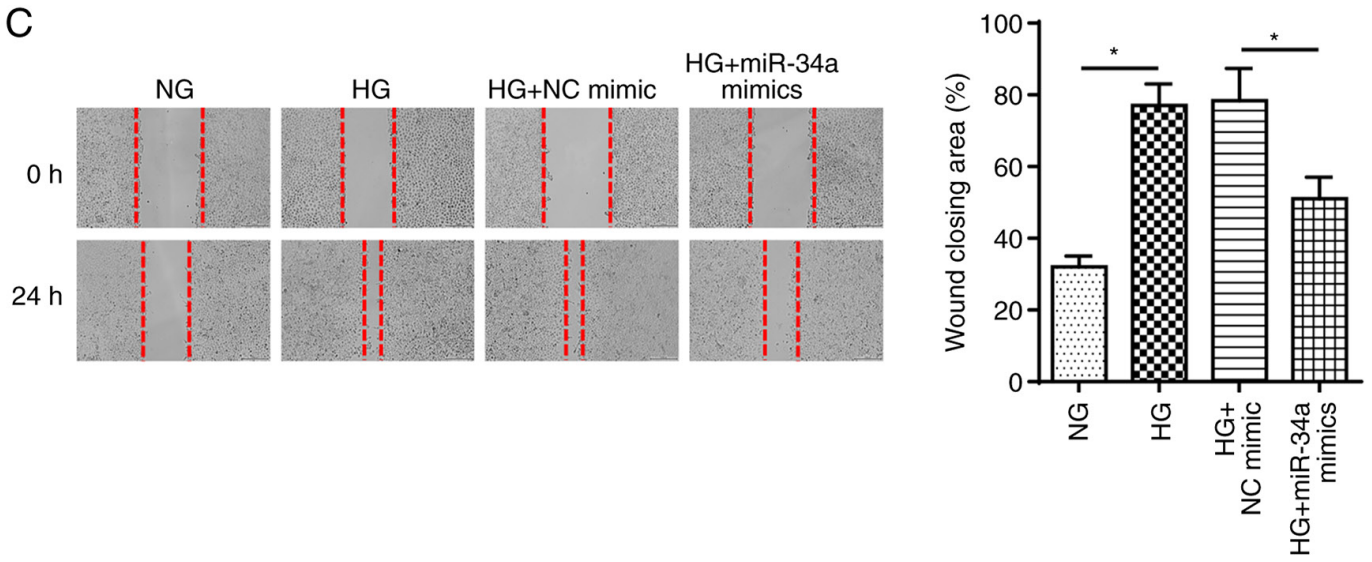

D
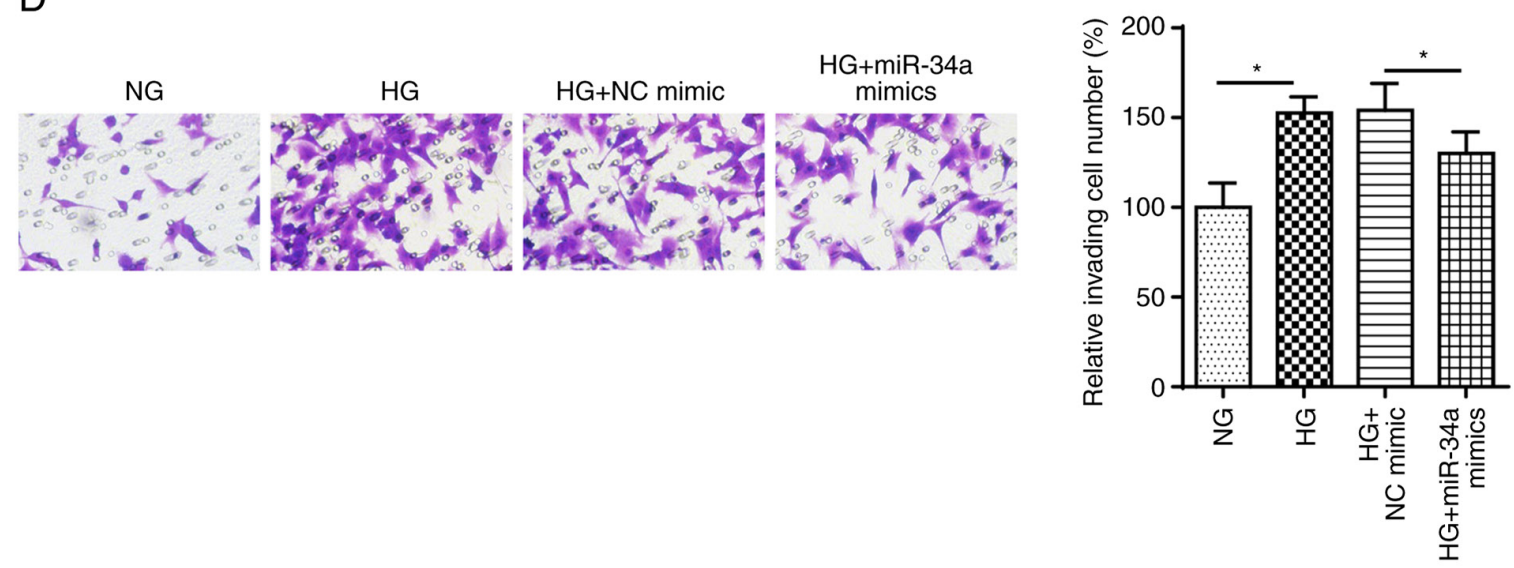

$E$
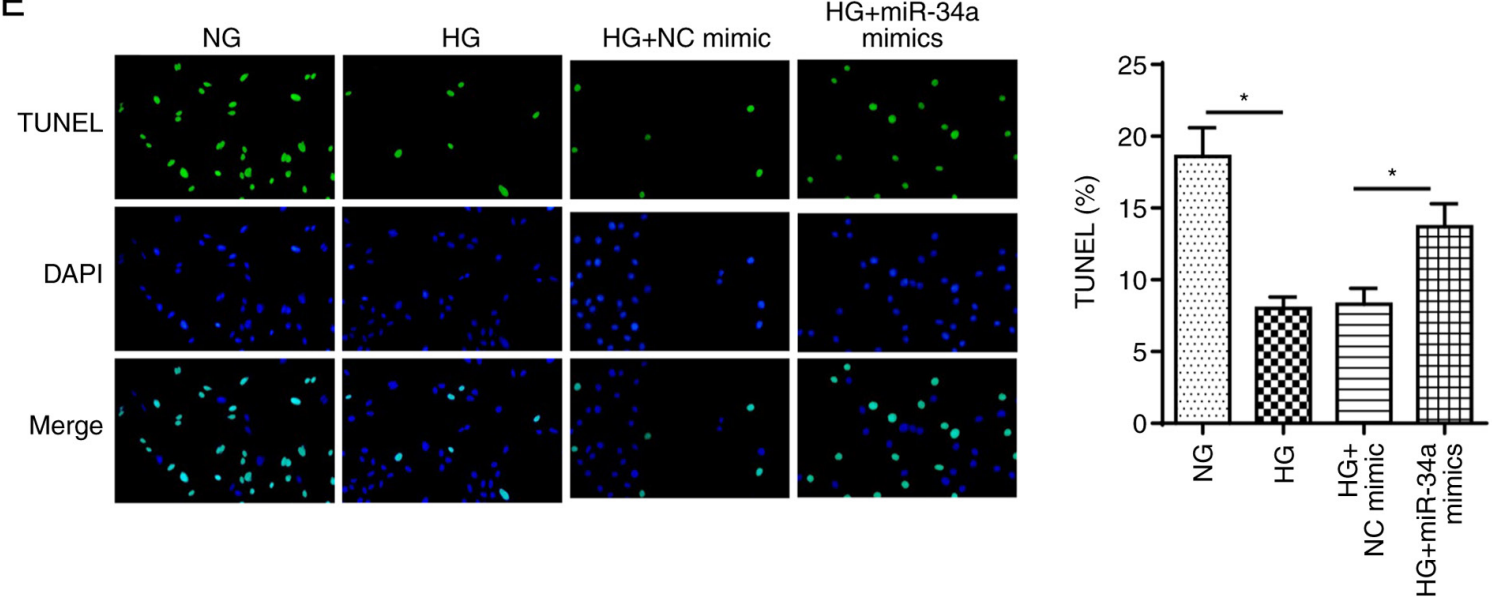

Figure 3. miR-34a overexpression partially inhibits the HG-mediated effects on proliferation, migration and invasion in lens epithelial cells. (A) Reverse transcription-quantitative PCR showed the relative miR-34a expression in SRA01/04 transfected with miR-34a mimics. "P $<0.05$ vs. NC mimics. (B) MTT, (C) wound healing and (D) Transwell assays were used to analyze the proliferation, migration and invasion of SRA01/04 cells transfected with miR-34a mimics or NC mimics after treatment of HG. (E) TUNEL assay determined the apoptosis in SRA01/04 cells transfected with miR-34a mimics or NC mimics after treatment of HG. $n=3$. "P<0.05. miR, microRNA; NG, normal glucose; HG, high glucose; NC, negative control. 

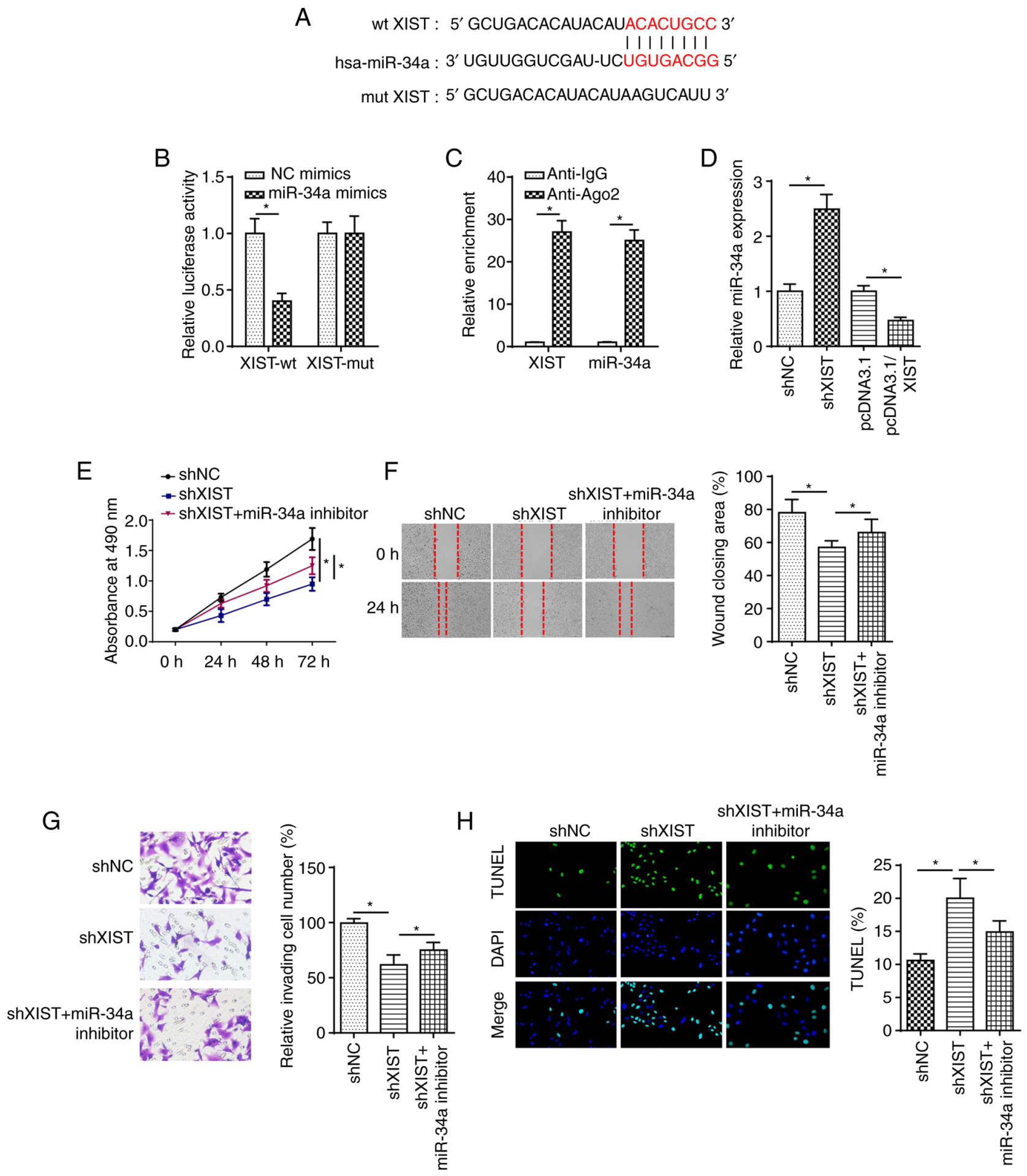

Figure 4. XIST knockdown modulates diabetic cataracts progression by targeting miR-34a in HG-induced lens epithelial cells. (A) StarBase website was used to predict the binding site between XIST and miR-34a. (B) Luciferase reporter assay showed luciferase activity of XIST-wt or XIST-mut in SRA01/04 cells transfected with NC mimics or miR-34a mimics. (C) RIP assay was performed to determine the enrichment of XIST and miR-34a in Anti-IgG and Anti-Ago2. (D) Reverse transcription-quantitative PCR showed the relative miR-34a expression in SRA01/04 cells transfected with pcDNA3.1, pcDNA3.1/XIST, shNC or shXIST. (E) MTT, (F) wound healing and (G) Transwell assays were used to analyze the proliferation, migration and invasion of SRA01/04 cells transfected with shNC, shXIST or shXIST + miR-34a inhibitor after treatment of HG. (H) TUNEL assay determined the apoptosis in SRA01/04 cells transfected with shNC, shXIST or shXIST + miR-34a inhibitor after treatment of HG. $n=3$. "P<0.05. XIST, X-inactive specific transcript; miR, microRNA; wt, wild-type; mut, mutant; sh, short hairpin RNA; NG, normal glucose; HG, high glucose; NC, negative control.

XIST deletion modulates DC progression by targeting miR-34a in HG-treated LECs. Subsequently, it was explored whether XIST modulates DC progression through miR-34a. As shown in Fig. 4A, the binding sequences of XIST and
miR-34a were predicted using starBase. Next, dual-luciferase reporter assay revealed that miR-34a overexpression repressed the luciferase activity of the XIST-wt group, while that of the XIST-mut group was unchanged (Fig. 4B). In addition, RIP 

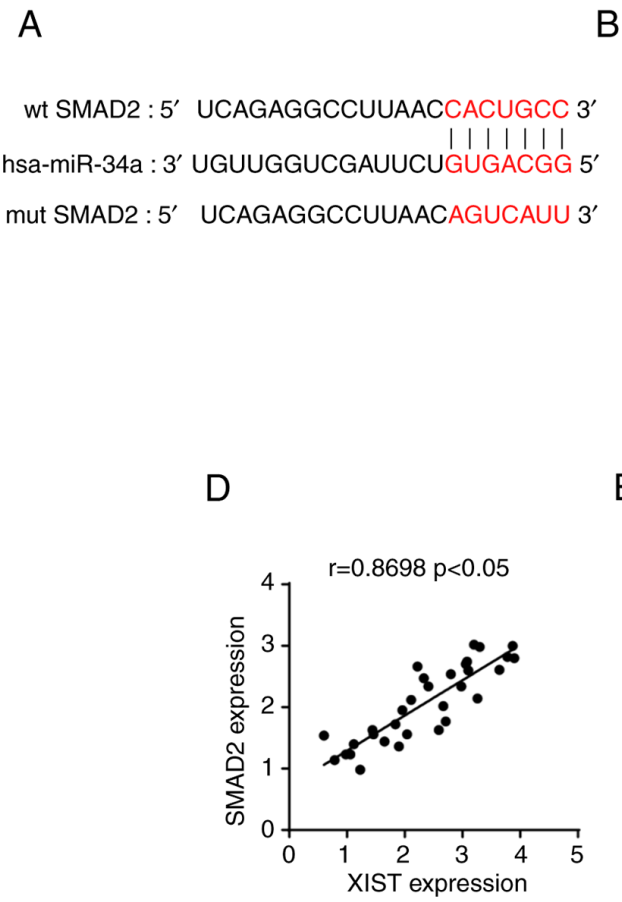

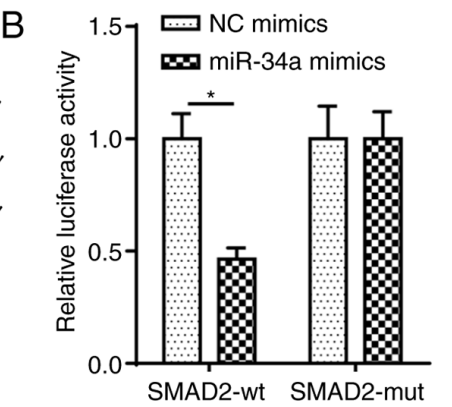

C

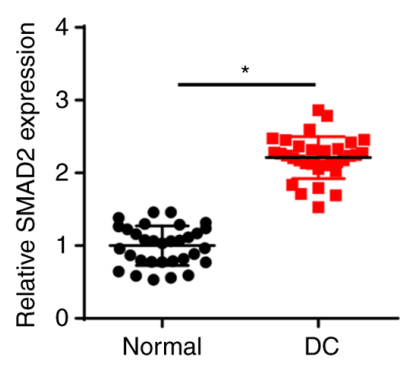

E

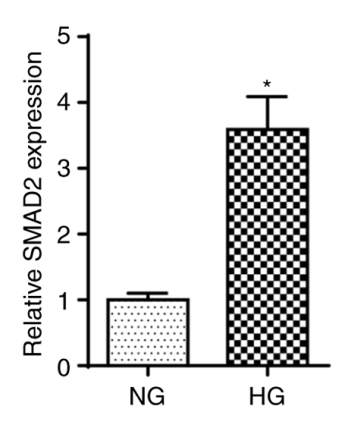

$\mathrm{F}$

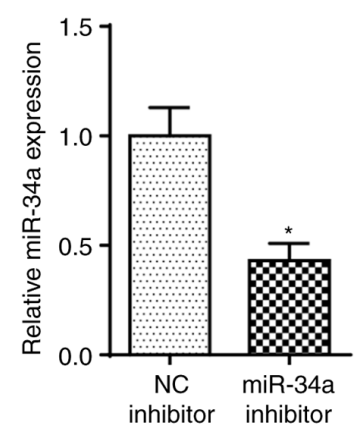

G

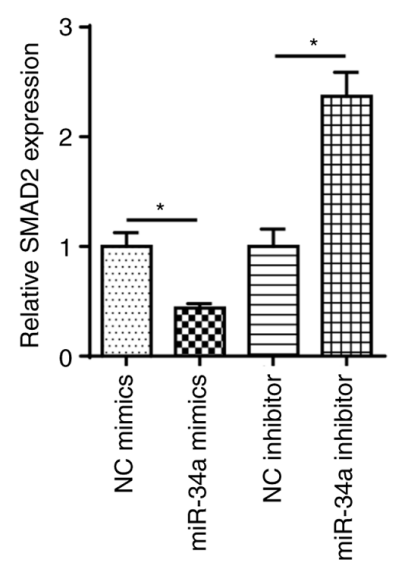

$\mathrm{H}$

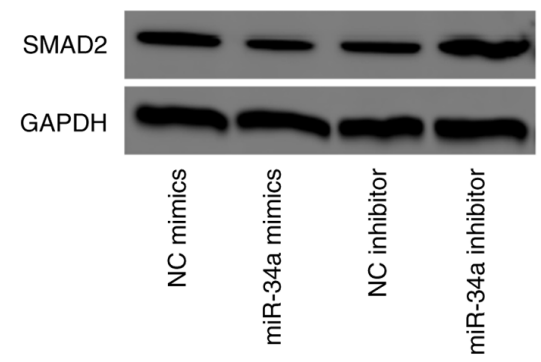

Figure 5. SMAD2 is a target of miR-34a. (A) StarBase website was used to predict the binding site between miR-34a and SMAD2. (B) Luciferase reporter assay showed luciferase activity of SMAD2-wt or SMAD2-mut in SRA01/04 cells transfected with NC mimics or miR-34a mimics. *P<0.05. (C) RT-qPCR showed the relative SMAD2 expression in DC tissues and normal samples. ${ }^{*} \mathrm{P}<0.05$. (D) Pearson's correlation analysis showed the correlation between $\mathrm{XIST}$ and $\mathrm{SMAD} 2$ expression in DC tissues. (E) RT-qPCR showed the relative SMAD2 expression in SRA01/04 cells treated with HG. * $<<0.05$ vs. NG group. (F) RT-qPCR showed the expression of miR-34a in SRA01/04 cells transfected with NC inhibitor and miR-34a inhibitor. "P<0.05 vs. NC inhibitor. (G and H) RT-qPCR and western blot assays showed the relative SMAD2 expression in SRA01/04 cells transfected with NC mimics or miR-34a mimics and NC inhibitor or miR-34a inhibitor. $n=3$. * $<<0.05$. SMAD2, SMAD family member 2; miR, microRNA; wt, wild-type; mut, mutant; NC, negative control; RT-qPCR, reverse transcription-quantitative PCR; DC, diabetic cataracts; XIST, X-inactive specific transcript; NG, normal glucose; HG, high glucose.

assay discovered that XIST and miR-34a enrichment was increased in LECs cells, which confirmed that XIST could combine with miR-34a (Fig. 4C). Furthermore, RT-qPCR demonstrated that the expression of miR-34a was reduced by XIST overexpression in SRA01/04 cells and enhanced by XIST knockdown (Fig. 4D). Functional assay suggested that miR-34a deficiency rescued the repressive effects of XIST knockdown on the proliferation, migration and invasion of HG-stimulated cells (Fig. 4E-G). Furthermore, XIST silencing induced cell apoptosis, which was abrogated by miR-34a deletion in HG-treated SRA01/04 cells (Fig. 4H). Therefore, the findings suggested that miR-34a is sponged by XIST and participates in the regulation of XIST during DC progression.
SMAD2 is a target of miR-34a. To explore the downstream mechanism of miR-34a in DC progression, the putative binding sites of miR-34a and SMAD2 were predicted using the starBase website (Fig. 5A). Next, the luciferase reporter assay revealed that miR-34a overexpression inhibited the luciferase activity of SMAD2-wt, but did not change that of SMAD2-mut (Fig. 5B). SMAD2 expression in DC tissues was elevated and was likely correlated with that of XIST (Fig. 5C and D). Furthermore, a higher expression of SMAD2 was observed in HG-treated SRA01/04 cells compared with NG group cells (Fig. 5E). RT-qPCR indicated that miR-34a expression was decreased in SRA01/04 cells transfected with miR-34a inhibitor (Fig. 5F). In addition, the mRNA and protein expression of SMAD2 in 
A

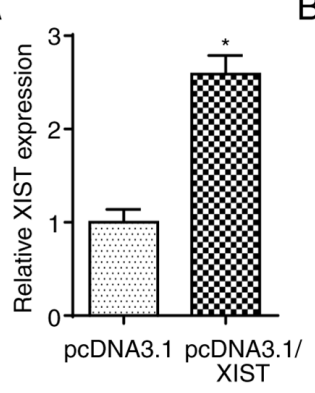

B

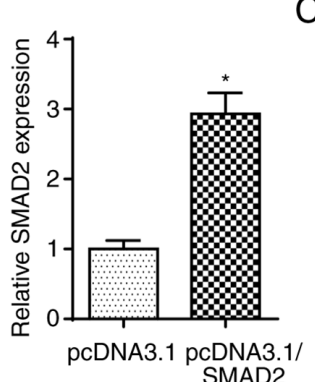

C

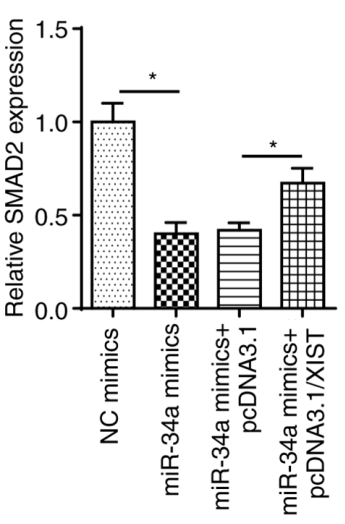

D

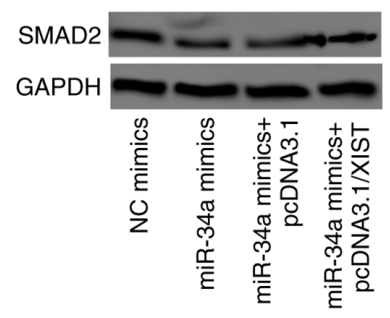

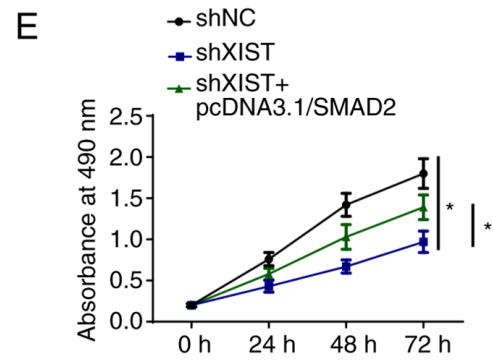

$\mathrm{F}$

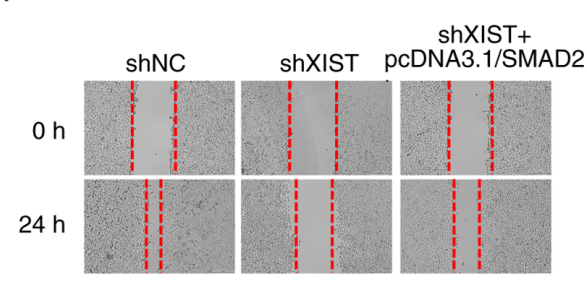

G

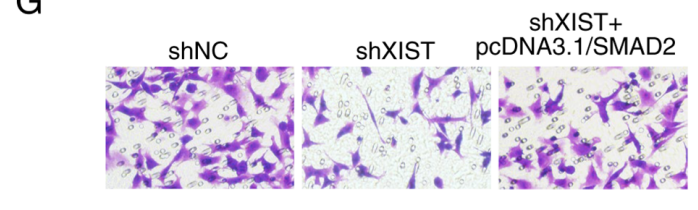

$\mathrm{H}$

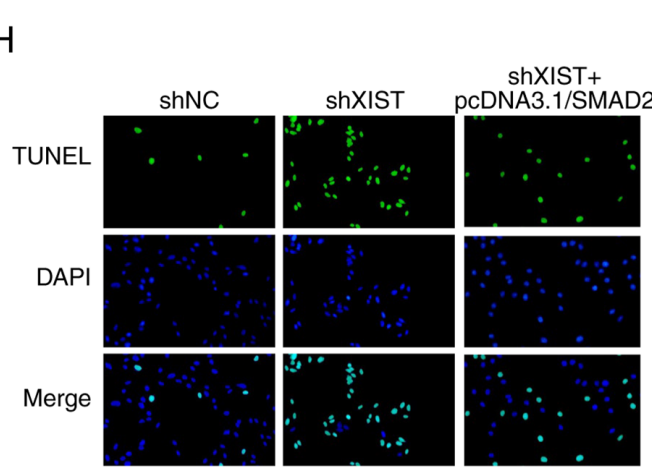

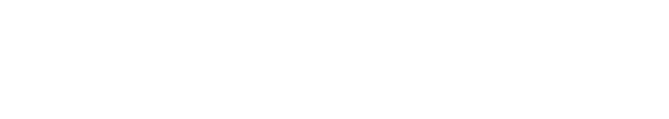
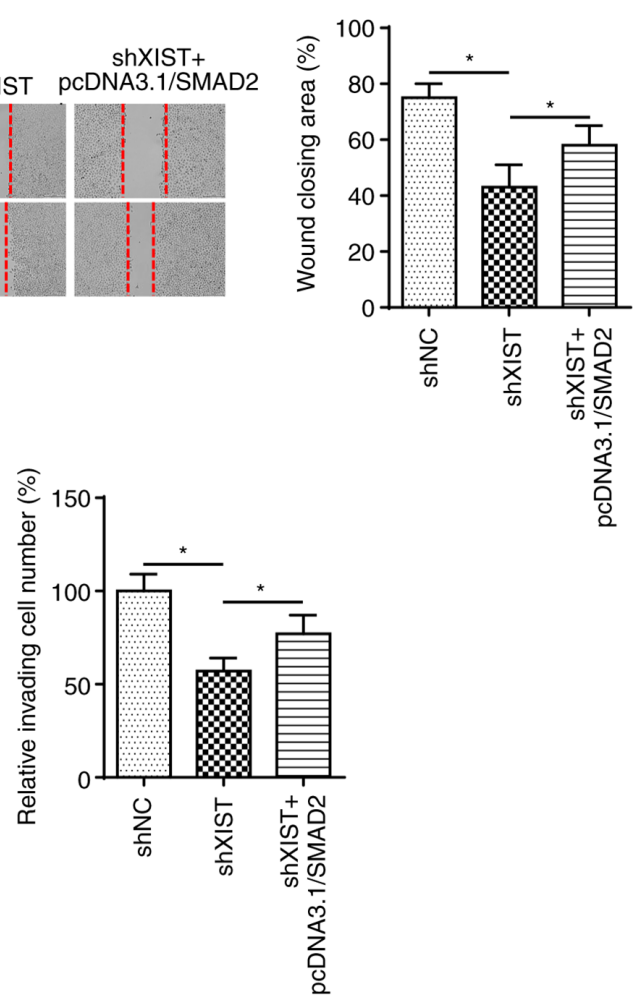

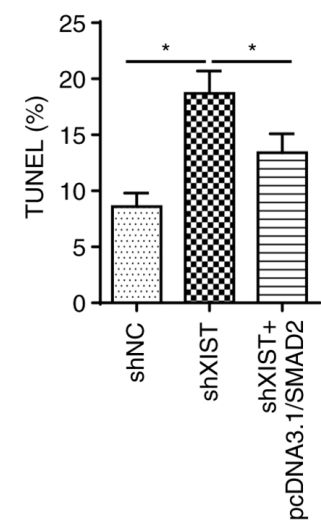

Figure 6. XIST accelerates diabetic cataracts progression through SMAD2 by sponging miR-34a in HG-treated lens epithelial cells. (A) RT-qPCR showed the expression of XIST in SRA01/04 cells transfected with pcDNA3.1 and pcDNA3.1/XIST. (B) RT-qPCR showed the expression of SMAD2 in SRA01/04 cells transfected pcDNA3.1 and pcDNA3.1/SMAD2. (C and D) RT-qPCR and western blot assays determined SMAD2 expression in SRA01/04 cells transfected with NC mimics, miR-34a mimics, miR-34a mimics + pcDNA3.1 and miR-34a mimics + pcDNA3.1/XIST. (E) MTT, (F) wound healing and (G) Transwell assays were used to analyze the proliferation, migration and invasion of SRA01/04 cells transfected with shNC, shXIST and shXIST + pcDNA3.1/SMAD2 after treatment of HG. (H) TUNEL assay determined the apoptosis in SRA01/04 cells transfected with shNC, shXIST and shXIST + pcDNA3.1/SMAD2 after treatment of HG. $n=3$. "P<0.05. XIST, X-inactive specific transcript; SMAD2, SMAD family member 2; miR, microRNA; HG, high glucose; RT-qPCR, reverse transcription-quantitative PCR; NC, negative control; sh, short hairpin RNA. 
SRA01/04 cells was decreased by miR-34a overexpression and increased by miR-34a knockdown (Fig. 5G and H). In combination, these findings indicated that miR-34a may target SMAD2 in SRA01/04 cells.

XIST accelerates DC progression through SMAD2 by sponging miR-34a in HG-treated LECs. Firstly, RT-qPCR showed that XIST and SMAD2 expression levels were significantly increased in SRA01/04 cells transfected with XIST and SMAD2 overexpression plasmids, respectively (Fig. 6A and B). The mRNA and protein expression levels of SMAD2 were decreased by miR-34a overexpression, which was rescued by XIST overexpression in HG-treated cells (Fig. 6C and D). To determine whether XIST mediated DC progression by modulating SMAD2, SRA01/04 cells were transfected with shNC, shXIST and shXIST + pcDNA3.1/SMAD2. Functional assays revealed that XIST silencing inhibited cell proliferation, migration and invasion in HG-stimulated SRA01/04 cells, but these effects were abrogated by SMAD2 overexpression (Fig. 6E-G). Furthermore, XIST silencing promoted HG-stimulated SRA01/04 cell apoptosis, which was reversed by SMAD2 overexpression (Fig. $6 \mathrm{H}$ ). In combination, these findings indicated that XIST may contribute to DC development by modulating SMAD2 in HG-treated LECs.

\section{Discussion}

DC is an early ocular complication in diabetic patients and one of the main causes of blindness (20). Certain studies have indicated that lncRNAs affect multiple biological processes in DC $(21,22)$. Therefore, understanding the function of IncRNAs in LECs under HG conditions may enable the discovery of novel targets for DC treatment. Previous studies indicated that enhanced proliferation, migratory capacity, invasiveness and resistance to apoptosis contribute to the occurrence of cataracts (23). Therefore, the present study investigated the role of XIST in regulating cell proliferation, migration and invasion during DC development. Collectively, these findings demonstrated that XIST regulated SMAD2 expression to participate in the occurrence and development of DC by sponging miR-34a.

XIST dysregulation has been observed in multiple biological processes, such as cell proliferation, invasion and apoptosis $(24,25)$. XIST has been reported to facilitate retinoblastoma progression by sponging miR-101 to regulate the levels of zinc finger E-box binding homeobox 1 and 2 (26). In addition, XIST knockdown repressed retinoblastoma development through the miR-124/STAT3 axis (27). In the present study, XIST expression was found to be increased in posterior capsular tissues and HG-stimulated SRA01/04 cells. XIST knockdown attenuated cell proliferation, migration and invasion, and induced apoptosis in HG-treated LECs. These findings suggested that XIST and miR-34a may represent promising targets for DC treatment.

Extensive evidence has demonstrated that XIST may serve as a ceRNA for miRNAs to regulate various diseases. For example, IncRNA XIST facilitated cell proliferation and invasion by targeting miR-137 to upregulate paxillin in non-small cell lung cancer (28). IncRNA XIST accelerated extracellular matrix degradation by acting as a ceRNA of miR-1277-5p in osteoarthritis (29). A previous study showed that miR-34a could restrain LEC proliferation and migration by reducing c-Met (30). In addition, Han et al (31) reported that miR-34a suppressed the EMT of LECs by targeting notch receptor 1 . In the present study, miR-34a was found to be downregulated in DC tissues and HG-stimulated SRA01/04 cells, and miR-34a overexpression in HG-treated LECs attenuated DC development. Furthermore, miR-34a deficiency reversed the effects of XIST deletion on cell viability, migration, invasion and apoptosis in HG-treated cells.

The nuclear translocation of SMAD2 has been confirmed as a crucial regulator of cell viability, migration and EMT $(32,33)$. The present study confirmed that SMAD2 was the downstream target of miR-34a. In addition, SMAD2 was also found to be upregulated in DC tissues and HG-stimulated SRA01/04 cells. XIST knockdown suppressed cell proliferation, migration and invasion and accelerated apoptosis in HG-treated SRA01/04 cells. However, these effects were weakened by SMAD2 overexpression. In combination, the results of the present study demonstrated that XIST modulated DC development by regulating SMAD2 in HG-treated SRA01/04 cells.

In conclusion, this study revealed a novel mechanism of XIST in DC progression. XIST facilitated cell proliferation, migration and invasion, and decreased apoptosis in HG-treated LECs through the miR-34a/SMAD2 axis, providing a novel biomarker for DC treatment. However, this study was not without its limitations. XIST expression was only examined in HG-treated LECs and DC tissues. Further studies detecting XIST levels in LECs from diabetic patients without cataracts are required.

\section{Acknowledgements}

Not applicable.

\section{Funding}

No funding was received.

\section{Availability of data and materials}

The datasets used and/or analyzed during the current study are available from the corresponding author on reasonable request.

\section{Authors' contributions}

$\mathrm{CW}$ and SZ designed the present study. CW, RZ and SZ performed the experiments. $C W$ and $R Z$ analyzed the data and prepared the figures. CW and SZ drafted the initial manuscript. All have authors read and approved the final manuscript. CW and SZ confirm the authenticity of all the raw data. All authors have read and approved the final manuscript.

\section{Ethics approval and consent to participate}

This study was approved by the Ethics Committee of Shandong Zaozhuang Municipal Hospital (Zaozhuang, China) and all participants signed informed consent forms prior to surgery. 


\section{Patient consent for publication}

Not applicable.

\section{Competing interests}

The authors declare that they have no competing interests.

\section{References}

1. McCarty CA and Taylor HR: Recent developments in vision research: Light damage in cataract. Invest Ophthalmol Vis Sci 37: 1720-1723, 1996.

2. Pollreisz A and Schmidt-Erfurth U: Diabetic cataract-pathogenesis, epidemiology and treatment. J Ophthalmol 2010: 608751, 2010.

3. Harding JJ, Egerton M, van Heyningen R and Harding RS: Diabetes, glaucoma, sex, and cataract: Analysis of combined data from two case control studies. Br J Ophthalmol 77: 2-6, 1993.

4. Kahn HA, Leibowitz HM, Ganley JP, Kini MM, Colton T, Nickerson RS and Dawber TR: The Framingham Eye Study. II. Association of ophthalmic pathology with single variables previously measured in the framingham heart study. Am J Epidemiol 106: 33-41, 1977.

5. Martinez G and de Iongh RU: The lens epithelium in ocular health and disease. Int J Biochem Cell Biol 42: 1945-1963, 2010.

6. Wang Y, Zhang G, Kang L and Guan H: Expression profiling of DNA methylation and transcriptional repression associated genes in lens epithelium cells of age-related cataract. Cell Mol Neurobiol 37: 537-543, 2017.

7. Mathieu EL, Belhocine M, Dao LT, Puthier D and Spicuglia S: Functions of lncRNA in development and diseases. Med Sci (Paris) 30: 790-796, 2014 (In French).

8. Gong W, Zhu G, Li J and Yang X: lncRNA MALAT1 promotes the apoptosis and oxidative stress of human lens epithelial cells via p38MAPK pathway in diabetic cataract. Diabetes Res Clin Pract 144: 314-321, 2018

9. Yang J, Zhao S and Tian F: SP1-mediated 1ncRNA PVT1 modulates the proliferation and apoptosis of lens epithelial cells in diabetic cataract via miR-214-3p/MMP2 axis. J Cell Mol Med 24: 554-561, 2020.

10. Dong Y, Wan G, Peng G, Yan P, Qian C and Li F: Long non-coding RNA XIST regulates hyperglycemia-associated apoptosis and migration in human retinal pigment epithelial cells. Biomed Pharmacother 125: 109959, 2020.

11. Wang Q: XIST silencing alleviated inflammation and mesangial cells proliferation in diabetic nephropathy by sponging miR-485. Arch Physiol Biochem: Jul 15, 2020 (Epub ahead of print). doi: 1 0.1080/13813455.2020.1789880.

12. Bartel DP: MicroRNAs: Genomics, biogenesis, mechanism, and function. Cell 116: 281-297, 2004.

13. Zhang L, Cheng $\mathrm{R}$ and Huang $\mathrm{Y}$ : miR-30a inhibits BECN1-mediated autophagy in diabetic cataract. Oncotarget 8 77360-77368, 2017

14. Zeng K, Feng QG, Lin BT, Ma DH and Liu CM: Effects of microRNA-211 on proliferation and apoptosis of lens epithelia cells by targeting SIRT1 gene in diabetic cataract mice. Biosci Rep 37: BSR20170695, 2017.

15. Xiang W, Lin H, Wang Q, Chen W, Liu Z, Chen H, Zhang H and Chen W: miR34a suppresses proliferation and induces apoptosis of human lens epithelial cells by targeting E2F3. Mol Med Rep 14: 5049-5056, 2016.

16. Li H, Song H, Yuan X, Li J and Tang H: miR-30a reverses TGF- $\beta 2$-induced migration and EMT in posterior capsular opacification by targeting Smad2. Mol Biol Rep 46: 3899-3907, 2019.

17. Livak KJ and Schmittgen TD: Analysis of relative gene expression data using real-time quantitative PCR and the 2(-Delta Delta C(T)) method. Methods 25: 402-408, 2001.
18. Li JH, Liu S, Zhou H, Qu LH and Yang JH: starBase v2.0 Decoding miRNA-ceRNA, miRNA-ncRNA and protein-RNA interaction networks from large-scale CLIP-Seq data. Nucleic Acids Res 42 (Database Issue): D92-D97, 2014.

19. Yang JH, Li JH, Shao P, Zhou H, Chen YQ and Qu LH: starBase: A database for exploring microRNA-mRNA interaction maps from Argonaute CLIP-Seq and Degradome-Seq data. Nucleic Acids Res 39 (Database Issue): D202-D209, 2011.

20. Zhou Y, Li L, Li S, Li S, Zhao M, Zhou Q, Gong X, Yang J and Chang J: Autoregenerative redox nanoparticles as an antioxidant and glycation inhibitor for palliation of diabetic cataracts. Nanoscale 11: 13126-13138, 2019.

21. Ye W, Ma J, Wang F, Wu T, He M, Li J, Pei R, Zhang L, Wang Y and Zhou J: IncRNA MALAT1 regulates miR-144-3p to facilitate epithelial-mesenchymal transition of lens epithelial cells via the ROS/NRF2/Notch1/Snail pathway. Oxid Med Cell Longev 2020: 8184314, 2020.

22. Li Y, Jiang SH, Liu S and Wang Q: Role of lncRNA NEAT1 mediated by YY1 in the development of diabetic cataract via targeting the microRNA-205-3p/MMP16 axis. Eur Rev Med Pharmacol Sci 24: 5863-5870, 2020

23. Wang $H$ and Zheng G: IncRNA NEAT1 promotes proliferation, migration, invasion and epithelial-mesenchymal transition process in TGF- $\beta 2$-stimulated lens epithelial cells through regulating the miR-486-5p/SMAD4 axis. Cancer Cell Int 20: 529, 2020.

24. Wang H, Li H, Yu Y, Jiang Q, Zhang R, Sun H, Xing W and Li Y: Long non-coding RNA XIST promotes the progression of esophageal squamous cell carcinoma through sponging miR-129-5p and upregulating CCND1 expression. Cell Cycle 20: 39-53, 2021

25. Wang N, He JX, Jia GZ, Wang K, Zhou S, Wu T and He XL: The lncRNA XIST promotes colorectal cancer cell growth through regulating the miR-497-5p/FOXK1 axis. Cancer Cell Int 20: 553 , 2020.

26. Cheng Y, Chang Q, Zheng B, Xu J, Li H and Wang R: IncRNA XIST promotes the epithelial to mesenchymal transition of retinoblastoma via sponging miR-101. Eur J Pharmacol 843: 210-216, 2019.

27. Hu C, Liu S, Han M, Wang Y and Xu C: Knockdown of lncRNA XIST inhibits retinoblastoma progression by modulating the miR-124/STAT3 axis. Biomed Pharmacother 107: 547-554, 2018.

28. Jiang $\mathrm{H}$, Zhang $\mathrm{H}, \mathrm{Hu} \mathrm{X}$ and $\mathrm{Li} \mathrm{W}$ : Knockdown of long non-coding RNA XIST inhibits cell viability and invasion by regulating miR-137/PXN axis in non-small cell lung cancer. Int J Biol Macromol 111: 623-631, 2018.

29. Wang T, Liu Y, Wang Y, Huang X, Zhao W and Zhao Z: Long non-coding RNA XIST promotes extracellular matrix degradation by functioning as a competing endogenous RNA of miR-1277-5p in osteoarthritis. Int J Mol Med 44: 630-642, 2019.

30. Feng D, Zhu N, Yu C and Lou D: MicroRNA-34a suppresses human lens epithelial cell proliferation and migration via downregulation of c-Met. Clin Chim Acta 495: 326-330, 2019.

31. Han R, Hao P, Wang L, Li J, Shui S, Wang Y, Ying M, Liu J, Tang X and Li X: MicroRNA-34a inhibits epithelial-mesenchymal transition of lens epithelial cells by targeting Notch1. Exp Eye Res 185: 107684, 2019.

32. Li H, Yuan X, Li J and Tang X: Implication of Smad 2 and Smad3 in transforming growth factor- $\beta$-induced posterior capsular opacification of human lens epithelial cells. Curr Eye Res 40: 386-397, 2015.

33. Li J, Tang $X$ and Chen $X$ : Comparative effects of TGF- $\beta 2 / \operatorname{Smad} 2$ and TGF- $\beta 2 / \mathrm{Smad} 3$ signaling pathways on proliferation, migration, and extracellular matrix production in a human lens cell line. Exp Eye Res 92: 173-179, 2011.

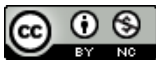

This work is licensed under a Creative Commons Attribution 4.0 International (CC BY-NC 4.0) License 